\title{
Competência cultural na atenção primária: algumas considerações
}

Cultural competence in primary care: some considerations

La competencia cultural en la atención primaria:algunas consideraciones

Renata Fiúza DAMASCENO ${ }^{(1)}$

Patrick Leonardo Nogueira da SILVA ${ }^{(2)}$

\footnotetext{
(1) Universidade Estadual de Montes Claros - UNIMONTES, Montes Claros, MG, Brasil.

(2) Prefeitura Municipal de Espinosa, Espinosa, Minas Gerais, Brasil..
}

Recebido:1 dez 2016 Revisado: 5 maio 2017 Aceito: 10 maio 2017

\section{Autor de}

correspondência:

Patrick Leonardo

Nogueira da Silva

patrick_mocesp70@hotmail .com

Conflito de interesses: Os autores declaram não haver nenhum interesse profissional ou pessoal que possa gerar conflito de interesses em relação a este manuscrito.

\section{Resumo}

O objetivo deste ensaio é apresentar algumas considerações sobre competência cultural na Atenção Primária à Saúde enquanto atributo responsável por qualificar suas ações. No Brasil, uma vez que a Atenção Primária à Saúde deve ser desenvolvida próxima da vida das pessoas, para produção do cuidado e da atenção integral, torna-se necessário que os profissionais que atuam nesse ponto de atenção considerem o sujeito em sua singularidade e inserção sociocultural, visto a grande influência que exercem os universos social e cultural sobre a adoção de comportamentos de prevenção ou de risco e sobre a utilização dos serviços de saúde. Sendo assim, é necessário, no âmbito do Sistema Único de Saúde, discutir estratégias para aumentar a competência cultural do profissional que atua na Atenção Primária à Saúde, independente do seu nível de escolaridade.

Descritores: Atenção primária à saúde, Competência cultural, Sistema Único de Saúde

\begin{abstract}
The objective of this essay is to present some considerations about cultural competence in Primary Health Care as an attribute responsible for qualifying its actions. In Brazil, since Primary Health Care must be developed close to people's lives, in order to produce care and integral care, it becomes necessary for professionals who work at this point of attention to consider the subject in its singularity and insertion Socio-cultural, since the social and cultural universes influence on the adoption of preventive or risk behaviors and on the use of health services. Therefore, it is necessary, within the Unified Health System, to discuss strategies to increase the cultural competence of professionals who work in Primary Health Care, regardless of their level of education.
\end{abstract}

Keywords: Primary Health Care; Cultural Competency; Unified Health System. 


\section{Resumen}

El objetivo de este trabajo es presentar algunas consideraciones sobre la competencia cultural en la atención primaria como atributo calificar responsables de sus acciones. En Brasil, ya que la atención primaria de salud deben desarrollarse cerca de las vidas de las personas, para proporcionar cuidado y atención integral, es necesario que los profesionales que trabajan en este punto de atención a revisar el tema en su unicidad e inserción socioculturales, ya que el impacto total de los universos sociales y culturales de la adopción de conductas de prevención o de riesgo y el uso de los servicios de salud. Por lo tanto, es necesario, en el marco del Sistema Nacional de Salud, discutir estrategias para aumentar la competencia cultural de los profesionales que trabajan en la atención primaria de salud, independientemente de su nivel de educación.

Palabras-claves:Atención primaria de salud, Competencia Cultural, Sistema Único de Salud

\section{Introdução}

No Brasil, a Atenção Primária à Saúde - APS se fortaleceu como eixo estruturador dos sistemas de saúde municipais, sendo que a mesma se apresenta nas mais diversas concepções e formas, espelhando a diversidade cultural e territorial brasileira.

A APS é reconhecidamente um componente-chave dos sistemas de saúde. Esse reconhecimento fundamenta-se nas evidencias de seu impacto na saúde e no desenvolvimento da população nos países que a adotaram como base para seus sistemas de saúde: melhores indicadores de saúde, maior eficiência no fluxo dos usuários dentro do sistema, tratamento mais efetivo de condições crônicas, maior eficiência do cuidado, maior utilização de práticas preventivas, maior satisfação dos usuários e diminuição das iniquidades sobre o acesso aos serviços e o estado geral de saúde. ${ }^{1,2}$

Atualmente, a principal estratégia de configuração da APS no Brasil é a saúde da família, que tem recebido importantes incentivos financeiros visando à ampliação da cobertura populacional e à reorganização da atenção à saúde no país. A saúde da família aprofunda os processos de territorialização e responsabilidade sanitária das equipes de saúde, compostas basicamente por médico generalista, enfermeiro, técnico ou auxiliar de enfermagem e agentes comunitários de saúde. ${ }^{3}$

Assim, uma vez que a APS no Brasil deve ser desenvolvida com o mais alto grau de descentralização e capilaridade, próxima da vida das pessoas, para produção do cuidado e da atenção integral torna-se necessário que os profissionais que atuam nesse ponto de 
atenção considerem o sujeito em sua singularidade e inserção sociocultural, visto a grande influência que exercem os universos social e cultural sobre a adoção de comportamentos de prevenção ou de risco e sobre a utilização dos serviços de saúde. ${ }^{4,5}$

Neste contexto, apesar dos trabalhos antropológicos na área de saúde terem aumentado progressivamente nos últimos anos, ainda são escassos estudos sobre competência cultural na APS, sendo que esse fato nos motivou a apresentar algumas considerações acerca do tema.

\section{Competência cultural: atributo da APS}

O pensar e o agir frente à saúde e à doença estão relacionados às experiências e vivências do indivíduo, família e profissional da saúde. Assim, tanto os modos de entender saúde como as práticas adotadas variam de indivíduo para indivíduo.

Os significados do adoecer e a percepção da doença para as pessoas atendidas na Atenção Primária são frequentemente pouco considerados, compreendidos e apreendidos pelos profissionais de saúde que atuam nesse ponto de atenção. Dessa forma, as orientações e ações educativas são realizadas considerando apenas as crenças dos profissionais, o que nem sempre está em consonância com o universo sócio-cultural do usuário. Esse fato é gerador de diversos dilemas e dificuldades na relação profissionalusuário e uma das causas da baixa adesão à terapêutica recomendada. ${ }^{6,7}$

As informações culturais têm sido, na maioria das vezes, consideradas irrelevantes para as intervenções preventivas e terapêuticas na área da saúde. ${ }^{8}$ Em geral, são tidas como essenciais unicamente àquelas referentes ao diagnóstico biomédico. Todos os outros dados, em particular aqueles referentes ao impacto dos fatores sociais e culturais, são avaliados como acessórios. ${ }^{9}$

Porém, tanto os cidadãos do mundo em desenvolvimento, como os dos países ricos, não estão apenas à procura de competência técnica: também querem que os profissionais de saúde sejam compreensivos, respeitadores e dignos de confiança. ${ }^{10}$ 
Querem cuidados de saúde organizados em torno das suas necessidades, que respeitem as suas crenças e que sejam sensíveis à situação particular da sua vida. Não querem ser considerados como meros alvos de programas de controle de doenças. Em países ricos e pobres, as pessoas querem mais assistência de saúde do que intervenções. Cada vez mais, há um reconhecimento de que a resolução dos problemas de saúde deveria levar em consideração o contexto sócio-cultural das famílias e das comunidades em que eles ocorrem. $^{11}$

Assim, observa-se a necessidade de aumentar a competência cultural dos profissionais de saúde que atuam na APS. Mas na verdade, o que é competência cultural? Nos últimos anos, o conceito de competência cultural tornou-se popular entre os planejadores de saúde, bem como entre médicos e enfermeiros, especialmente na América do Norte. Isso resultou, em boa parte, do aumento da diversidade cultural e étnica da população e da necessidade de melhorar a comunicação com as minorias e os imigrantes, bem como de melhorar a qualidade de seus cuidados médicos. ${ }^{12}$

De acordo com o Office of Minority Health- $\mathrm{OMH}$ do governo dos Estados Unidos, a competência cultural e linguística implica uma capacidade dos fornecedores e das organizações de cuidados em saúde de compreender e responder efetivamente às necessidades culturais e linguísticas, trazidas pelos pacientes às situações de cuidados de saúde. ${ }^{13}$ Assim, a competência cultural tem várias dimensões:

1. Melhorar a sensibilidade dos profissionais de saúde às crenças culturais, práticas, expectativas e origens de seus pacientes e suas comunidades (como as crenças sobre as origens da má saúde, o papel maior da família em tomar decisões relacionadas à saúde ou a preferência que têm algumas pacientes mulheres por serem examinadas somente por um profissional de saúde do sexo feminino). ${ }^{12}$

2. Melhorar o acesso aos cuidados de saúde, eliminando as barreiras estruturais aos cuidados de saúde de qualidade para as minorias (fornecendo intérpretes, dietas hospitalares de acordo com crenças religiosas, períodos de espera mais curtos para 
consultas e materiais de educação em saúde culturalmente apropriados). Também incluídas aqui estão as dificuldades que as minorias encontram em obter consultas médicas regulares ou em ser encaminhadas a um especialista. ${ }^{14}$

3. Reduzir as barreiras organizacionais, como o pequeno número de profissionais de saúde, administradores e elaboradores de políticas selecionados dentre comunidades de minorias, os quais poderiam planejar serviços de saúde mais apropriados culturalmente. ${ }^{14}$

Apesar da utilidade óbvia do conceito de competência cultural, é importante notar que ela não é um substituto para competência clínica. Ser culturalmente competente não necessariamente significa que se é um bom médico ou enfermeiro. A competência clínica é uma importante habilidade complementar que deve ser adquirida por todos os profissionais de saúde, seja qual for o contexto em que trabalhem. Além de lidar com uma população diversificada, essa habilidade é essencial porque as relações entre os profissionais de saúde e seus pacientes - de qualquer origem - muitas vezes podem ser descritas como um choque de culturas em si. $^{13}$

Na APS, a competência cultural juntamente com a orientação centrada na família e a orientação comunitária são consideradas atributos derivados que qualificam suas ações por garantirem um alto nível de alcance das qualidades exclusivas e fundamentais da APS.

Neste contexto, a competência cultural envolve o reconhecimento das necessidades especiais das subpopulações que podem não estar em evidência devido a características étnicas, raciais ou outras características culturais especiais. Se a integralidade, particularmente o aspecto relacionado com o reconhecimento do problema, for bem alcançada, estas necessidades especiais deveriam ser reconhecidas e abordadas no projeto da variedade de serviços e o quão bem eles são aplicados. ${ }^{1}$

Assim, uma série de benefícios pode surgir do aumento da competência cultural do profissional de saúde. Pode-se melhorar não somente a comunicação médico-paciente, mas também a satisfação do paciente e a adesão (como menos faltas a consultas). Pode 
haver também um impacto positivo no diagnóstico e no tratamento das doenças, bem como uso adequado dos recursos médicos. Além disso, em longo prazo, pode ocorrer a redução de disparidades na saúde entre as minorias e a população majoritária. ${ }^{13,15,16}$

\section{Considerações finais}

Desta forma, é necessário no âmbito do Sistema Único de Saúde (SUS) discutir a formação dos profissionais que atuam na APS, bem como propor estratégias para aumentar a competência cultural desses profissionais, independente do seu nível de escolaridade, já que a falta dessa preparação pode ainda ser agravada por conflitos interculturais, pela estratificação social, pela discriminação e pela estigmatização. As consequências disso são que o considerável potencial das pessoas virem a contribuir para a sua própria saúde por meio da adequação dos seus estilos de vida, dos seus comportamentos e da autoajuda, e a possibilidade de otimização do aconselhamento profissional às suas circunstâncias de vida, são subutilizadas.

\section{Referências}

1. Starfield B. Atenção primária: equilíbrio entre necessidades de saúde, serviços e tecnologia. Brasília, DF: UNESCO, Ministério da Saúde; 2002. 726p.

2. Tasca, R., coord.A atenção à saúde coordenada pela APS: construindo as redes de atenção no SUS: contribuições para o debate. Brasília, DF: OPAS; 2011.

3. Matta GC, Morosini MVG. Atenção Primária à Saúde. Disponível em:http://www.sites.epsjv.fiocruz.br/dicionario/verbetes/ateprisau.html.

4. Brasil, Ministério da Saúde, Secretaria de Atenção à Saúde, Departamento de Atenção Básica. Política Nacional de Atenção Básica. Brasília, DF: MS, 2012. 110 p.

5. Taylor P, Chandiwana SK, Govere JM, Chombo F. Knowledge attitudes and practices in relation to schistosomiasis in a rural community. Social Sciences and Medicine. 1987;24:607-11.https://doi.org/10.1016/0277-9536(87)90065-7.

6. Fertonani HP. O desafio de construir um novo modelo assistencial em saúde: reflexões de trabalhadores de saúde do PSF de uma unidade de saúde [dissertação]. Florianópolis: Universidade Federal de Santa Catarina; 2003. 
7. Silva DMGV. Narrativas do viver com diabetes mellitus: experiências pessoais e culturais [Tese]. Florianópolis: Universidade Federal de Santa Catarina; 2000. 188p.

8. Good BJ, Good MJD. The meaning of symptoms: a cultural hermeneutic model for clinical practice. In Eisenberg, L., KleinmanA., editors. The Relevance of Social Science for Medicine.Dordrechet: Reideil Publishing; 1980. pp. 165-96.https://doi.org/10.1007/97894-009-8379-3_8.

9. Kleinman, A.Patients and healers in the context of cultures: an exploration of boderland between Anthropology and Psychiatry. Berkeley, Los Angeles: University of California Press.

10. Bossyns $P$, Van Lerberghe $W$. The weakest link: competence and prestige as constraints to referral by isolated nurses in rural Niger. Hum Resour Health. 2004, 2(1):18.https://doi.org/10.1186/1478-4491-2-1.

11. Cheragi-Sohi S.Bower P, Mead N, McDonald R, Whalley D, Roland M. What are the key attributes of primary care for patients? Building a conceptual map of patient preferences. Health Expect. 2006;9(3):275-84.

12. Like RC, Steiner RP, Rubel AJ. Recommended core curriculum guidelines on culturally sensitive and competent health care. Fam Med. 1996;28(4):291-7.

13. HelmanCG. Cultura, saúde e doença. São Paulo: Artmed; 2009. 432p.

14. Betancourt JR, Green AR, Carillo JE, Ananeh-Firempong O 2nd. Defining cultural competence: a practical framework for addressing racial/ethnic disparities in health and health care. Public Health Rep. 2003;118(4):293302.https://doi.org/10.1093/phr/118.4.293

15. Willems S, De Maesschalck S, Deveugele M, Derese A, De Maeseneer J. Socio-economic status of the patient and doctor-patient communication: does it make a difference. Patient Educ Couns. 2005;56(2):139-46.

16. Pan American Health Organization.Renewing primary health care in the Americas: a Position paper of the Pan American Health Organization. Washington, DC: PAHO; 2007. 48 p. Disponível em: http://iris.paho.org/xmlui/handle/123456789/31083. 


\section{Renata Fiúza Damasceno}

Doutoranda pelo Programa de Pós-Graduação em Ciências da Saúde - PPGCS da Universidade Estadual de Montes Claros - UNIMONTES. Enfermeira da Superintendência Regional de Saúde de Montes Claros SRS/MOC.

\section{Patrick Leonardo Nogueira da Silva}

Especialista em Enfermagem do Trabalho pela Faculdade de Guanambi (FG). Enfermeiro da Prefeitura Municipal de Espinosa. 